\title{
Fast and accurate exercise policies for Bermudan swaptions in the LIBOR market model
}

\author{
Patrik Karlsson ${ }^{\dagger, \uparrow}$, Shashi Jain ${ }^{\star}$ and Cornelis W. Oosterlee ${ }^{* \S}$ \\ ${ }^{*}$ CWI-Centrum Wiskunde \& Informatica \\ Amsterdam, The Netherlands \\ 'Department of Economics, Lund University \\ P. O. Box 7082, S-220 07 Lund, Sweden \\ ING, Amsterdam, The Netherlands \\ \$TU Delft, Delft Institute of Applied Mathematics \\ Delft, The Netherlands
}

Received: 5 June 2015; Accepted: 21 November 2015

Published: 20 May 2016

\begin{abstract}
This paper describes an American Monte Carlo approach for obtaining fast and accurate exercise policies for pricing of callable LIBOR Exotics (e.g., Bermudan swaptions) in the LIBOR market model using the Stochastic Grid Bundling Method (SGBM). SGBM is a bundling and regression based Monte Carlo method where the continuation value is projected onto a space where the distribution is known. We also demonstrate an algorithm to obtain accurate and tight lower-upper bound values without the need for nested Monte Carlo simulations.
\end{abstract}

Keywords: Applied mathematical finance; Bermudan swaptions; computational finance; derivative pricing models; interest rate modelling; LIBOR market model.

\section{Introduction}

A Bermudan receiver (payer) swaption (i.e., an Bermudan option on a interest rate swap) is today one of the most liquid and important options. It gives the owner the right - but not the obligation - to enter into a receiver (payer) interest rate swap at a discrete set of dates (exercise dates). This contrasts the European swaption,

Email address: "patrik.karlsson@ nek.lu.se 
which only can be exercised on a single exercise date and the American options, which can be exercised at any point before the maturity.

The LIBOR market model (LMM) is popular for modelling and pricing interest rate derivatives, see for instance Miltersen et al. (1997), Brace et al. (1997) and Jamshidian (1997). The LMM dynamics are specified as nonoverlapping sets of discretely compounded LIBOR rates. Its consistency with the market practice of pricing fixed-income derivatives allows for pricing to be reduced to standard market formulae such as, for example, the Black (1976) formula, and its ability to price securities that rely strongly on correlations between forward rates are reasons for its popularity. Pricing Bermudan swaptions in the LMM is a more complex problem than pricing corresponding European options. First, the holder of a Bermudan swaption is in a position where, at each exercise date, he needs to determine whether it is optimal to exercise the option or holding on to it. Second, due to the high dimensionality of LMM, ${ }^{1}$ only Monte Carlo methods are feasible for valuation of exotic fixed-income securities, such as Bermudan swaptions.

Pricing American-style derivatives via Monte Carlo simulation has been actively studied. The industrial standard Longstaff and Schwartz (2001) method, Least-Squares Monte Carlo (LSM), uses a regression to approximate the continuation value for a set of simulated paths. The fact that LSM is easy to implement, robust, and generates accurate lower bound Bermudan swaptions values for a careful choice of regression variables are reasons for its popularity. Lower bounds have moreover been studied in Andersen (1999) where a presimulation is performed to estimate a parametrized exercise policy which is then used in a larger simulation for valuating Bermudan swaptions. Generally, American Monte Carlo techniques as LSM (for lower bound values) are divided into two passes, a first and a second pass. In the first pass where the exercise strategy is estimated, the conditional discounted option values are projected onto basis functions of the state variables. The projected value is then used as the approximate continuation value, which is compared with the intrinsic value for determining the optimal exercise strategy. This is then followed by a second pass where the low-biased option values are obtained by simulating a new set of simulation paths, and exercising according to the sub-optimal exercise strategy obtained in the first pass.

To validate the pricing models and the lower bound values generated from the second pass, we need a third pass. These are referred as the upper bound values and the closer they are to the lower bounds, the better. This has previously been studied in Rogers (2002), Haugh and Kogan (2001) and Andersen and Broadie (2004), where the upper bound is approximated via a duality approach. Generally,

\footnotetext{
${ }^{1}$ E.g., modelling a Bermudan swaption on a swap with 10 year maturity and frequency 3 months requires about 40 Libor rates.
} 
upper bounds algorithms as e.g., in Andersen and Broadie (2004) require nested Monte Carlo simulation which makes it computationally expensive. The quality of the upper bound produced by the algorithm depends on the quality of the estimated exercise policy in the first pass, better policy gives tighter upper-lower bounds.

The stochastic grid bundling method (SGBM) was introduced in Jain and Oosterlee (2015) for the pricing of equity Bermudan options on geometric Brownian motions. They show that SGBM increases the efficiency of Monte Carlo simulation by reducing the variance of simulation estimates based on conditional expectations and the use of regression, as in Milstein and Tretyakov (2009). The method is based upon the stochastic grid method (SGM) by Jain and Oosterlee (2012a), LSM and the bundling approach by Tilley (1993). The idea behind SGBM is that for a large set of paths, neighbouring simulated paths will have similar continuation values and one can therefore perform local-averaging to compute a continuation value for grid points within a bundle using regression. The main difference between LSM and SGBM is that in SGBM one projects the option values onto a set of basis functions of the state variables where the distribution is analytically (or approximately) known, whereas in LSM the distribution is not taken into account.

This paper is more than a re-interpretation of Jain and Oosterlee (2015). First, since the interest rates are stochastic, compared to the fixed in Jain and Oosterlee (2015), the trivial expectations for the continuation values need to be carefully calculated. One of the interesting aspects of SGBM employed in the present paper is that because of a formulation in terms of an inner and outer expectation, for calculating the continuation value, we can benefit from the flexibility to use different pricing measures within the same problem. In the case of Bermudan swaptions it allows us to use the spot measure, useful for simulating paths, as well as the forward measure, which allows the discounting term to be taken out of the expectation and so gives rise to an analytic expression for the outer expectation. Second, we also present an efficient way of obtaining upper bound values for Bermudan swaptions in LMM by avoiding nested Monte Carlo simulations and therefore reduce the required computational time. Third, we demonstrate that SGBM gives more accurate results and is computationally more attractive than LSM.

The paper is organized as follows. Section 2 introduces notations, the general framework and formulates the Bermudan swaption pricing problem in the LMM. Section 3 describes LSM and the SGBM algorithm for pricing Bermudan swaptions (both a lower and an upper bound method). In Sec. 4, we present various numerical examples to illustrate the method and finally we conclude in Sec. 5. 


\section{Notation and General Framework}

In this section, we introduce notation, give a short introduction to the LMM and define the Bermudan swaption pricing. We follow the notation in Andersen and Piterbarg (2010).

\subsection{The LIBOR market model}

For the LMM, we start with a fixed discrete-tenor structure $0=T_{0}$ $<T_{1}<\cdots<T_{N}$. The intervals over the time horizon are given by $\tau_{n}=T_{n+1}-T_{n}$ and are typically three or six calendar months. Let $P\left(t, T_{n}\right)$ denote the time- $t$ price of a zero-coupon bond delivering one unit of currency at some maturity time $T_{n} \geq t$. The discrete LIBOR forward rate $L_{n}(t)$ with fixing date $T_{n}$ as seen at time $t$ is

$$
L_{n}(t)=\tau_{n}^{-1}\left(\frac{P\left(t, T_{n}\right)}{P\left(t, T_{n+1}\right)}-1\right), \quad N-1 \geq n \geq q(t),
$$

where $q(t)$ is the index function of the bond with the shortest maturity, defined as $T_{q(t)-1} \leq t<T_{q(t)}$. The price of the discounted bond maturing at time $T_{k}>t$ is then given by

$$
P\left(t, T_{n}\right)=P\left(t, T_{q(t)}\right) \prod_{n=q(t)}^{n-1} \frac{1}{1+\tau_{n} L_{n}(t)} .
$$

For the set of LIBOR rates $L(t)=\left(L_{q(t)}, L_{q(t)+1}, \ldots, L_{N-1}(t)\right)$, we choose to work under the spot LIBOR measure, denoted by $\mathbb{Q}^{B}$, in which the discrete money market account $B(t)$ is the numeraire, given by

$$
B(t)=P\left(t, T_{q(t)}\right) \prod_{n=0}^{q(t)-1}\left(1+\tau_{n} L_{n}(t)\right) .
$$

The no-arbitrage dynamics of the forward LIBOR rates $L_{n}(t)$ under the spot LIBOR measure $\mathbb{Q}^{B}$ for $n \geq q(t)$ are given by

$$
\begin{aligned}
d L_{n}(t) & =L_{n}(t) \lambda_{n}(t)^{\top}\left(\mu_{n}(t) d t+d W^{B}(t)\right), \\
\mu_{n}(t) & =\sum_{i=q(t)}^{n} \frac{\tau_{i} \lambda_{i}(t)}{1+\tau_{i} L_{i}(t)},
\end{aligned}
$$

where $W^{B}(t)$ is an $m$-dimensional Brownian motion under measure $\mathbb{Q}^{B}$ and $\lambda_{n}$ for $n \geq q(t)$, is a bounded $m$-dimensional deterministic function. Let $\mathbb{E}_{t}[\cdot]=$ $\mathbb{E}\left[\cdot \mid \mathcal{F}_{t}\right]$ be denoting the conditional expectation at time $t$ under the spot LIBOR 
measure and where $\mathcal{F}_{t}$ is the filtration at time $t$ generated by $W^{B}$. Then by standard arbitrage-free arguments the time- $t$ price of a security paying $V(T)$ at time $T$ is

$$
V(t)=\mathbb{E}_{t}\left[V(T) \frac{B(t)}{B(T)}\right] .
$$

Further details on the LMM, such as derivations of the bond equations, connection to HJM etc., is out of the scope of this paper and can be found in Andersen and Piterbarg (2010).

\subsection{Bermudan swaptions}

Given a lockout, i.e., a no-call period up to time $T_{1}$, the Bermudan swaption gives the holder the right, but not the obligation, on a set of fixing dates $T_{n}$ in $\mathcal{T}=\left\{T_{1}, T_{2}, \ldots, T_{m-1}\right\}$, for $m \leq N-1$, to enter into a fixed for floating swap with fixing date $T_{n}$ and last payment date $T_{m}$. The holder of a payer Bermudan will pay the fixed swap leg and receive the floating swap leg. If exercise at $T_{n}$ the payout is given by

$$
U\left(T_{n}\right)=\phi \mathcal{N} \sum_{i=n}^{m-1} \tau_{i} P\left(T_{n}, T_{i+1}\right)\left(L_{i}\left(T_{n}\right)-k\right),
$$

where $k$ is the fixed coupon, $\mathcal{N}$ the notional, and $\phi \in\{-1,+1\}$ is the payer or receiver factor ( +1 for payer swaption and -1 for a receiver swaption). The payoff is also equivalent to

$$
U\left(T_{n}\right)=\phi \mathcal{N} A_{n, m}\left(T_{n}\right)\left(S_{n, m}\left(T_{n}\right)-k\right),
$$

where $S_{n, m}(t)$ is the value of the fixed-for-floating swap with payments at times $T_{n+1}, \ldots, T_{m}$, see for instance Andersen and Piterbarg (2010, Chapter 19). The value of the forward swap rate $S(t)$ and swap annuity $A(t)$ at time $t$ are given by.

$$
S(t):=S_{n, m}(t)=\frac{P\left(t, T_{n}\right)-P\left(t, T_{m}\right)}{A_{n, m}(t)}, \quad A(t):=A_{n, m}(t)=\sum_{i=n}^{m-1} P\left(t, T_{i+1}\right) \tau_{i} .
$$

The present value $V\left(T_{0}\right)$ of a Bermudan swaption at time $T_{0}$ is the supremum taken over all discrete stopping times of all conditional expected discounted payoffs, that is

$$
\begin{aligned}
V\left(T_{0}\right) & =B\left(T_{0}\right) \sup _{\tau \in \mathcal{T}} \mathbb{E}_{0}\left[\frac{U(\tau)}{B(\tau)}\right] \\
& =B\left(T_{0}\right) \mathbb{E}_{0}\left[\frac{U\left(\tau^{*}\right)}{B\left(\tau^{*}\right)}\right],
\end{aligned}
$$


where $\tau * \in \mathcal{T}$ is the optimal stopping time taking values in the finite set of allowed discrete exercise dates $\mathcal{T}$. For the American swaption, the holder is allowed to exercise on any date within $\left[T_{1}, T_{m-1}\right]$. And for European swaption case, we have only one exercise date, i.e., $T_{1}=T_{m-1}$.

\section{Monte Carlo Simulation of Bermudan Swaptions}

In this section, we define the Bermudan option pricing problem, summarize SGBM, present a bundling algorithm suitable for the pricing of Bermudan swaptions in the LMM and discuss our implementation of the LSM and the upper and lower bounds via SGBM.

The present value $V(0)$ of a Bermudan swaption in (4) is usually solved via backward induction starting from the last exercise date $T_{m-1}$. The holder of the option receives $U\left(T_{n}\right)$ if the contract is exercised at time $T_{n}$. The option value at $V\left(T_{n}\right)$ at time $T_{n}$ is the maximum of the intrinsic value $U\left(T_{n}\right)$ and the conditional continuation value $H\left(T_{n}\right)$, that is

$$
V\left(T_{n}\right)=\max \left(U\left(T_{n}\right), H\left(T_{n}\right)\right),
$$

where $H\left(T_{m-1}\right)=0$. The conditional continuation value $H\left(T_{n}\right)$ is the conditional expected time $T_{n+1}$ option value given by,

$$
H\left(T_{n}\right)=B\left(T_{n}\right) \mathbb{E}_{T_{n}}\left[\frac{V\left(T_{n+1}\right)}{B\left(T_{n+1}\right)}\right] .
$$

The problem is solved by recursively repeating Eqs. (6) and (7) for each $T_{n}$ until we reach time $T_{0}$, where we find the value $V\left(T_{0}\right)$ of the contract.

As mentioned in the introduction, lower bound American Monte Carlo methods as LSM and SGBM are divided into two phases, a first and a second pass. In the first pass, the conditional discounted option values are projected onto basis functions of the state variables. The projected value is then used as the approximate continuation value, which is compared with the intrinsic value for determining the optimal exercise strategy. This is followed by a second pass where the low-biased option values are obtained by simulating a new set of simulation paths, and exercising according to the sub-optimal exercise strategy obtained in the first pass.

\subsection{The least squares method}

In the least squares method (LSM), the problem is solved by recursive value iteration, by the dynamic programming approach, starting from the last exercise date and working backwards as given by Eqs. (6) and (7). As pointed out by Clement et al. (2002), the main problem with dynamic programming is the evaluation of the conditional expectation. The LSM method is based on 
approximation of the conditional expectation of $H\left(T_{n}\right)$ at time $T_{n}$ by an ordinary least squares estimate,

$$
H\left(T_{n}\right)=\sum_{i=0}^{q} \beta_{i, n} \zeta_{i}\left(T_{n}\right),
$$

for a set of $q$ basis-functions $\zeta_{i}: \mathbb{R}^{d} \rightarrow \mathbb{R}, i=1,2, \ldots, q$, e.g., function of the underlying swap rates, and where $\beta_{i, n}$ are constants. The regression is usually performed using the simulated in-the-money paths and the basis functions are usually polynomials of the state variables. The optimal stopping time derived using this approximation, denoted by $\tau$, can be written as

$$
\tau_{n}=t_{n} \mathbf{1}\left\{H\left(T_{n}\right) \leq U\left(T_{n}\right)\right\}+t_{n+1} \mathbf{1}\left\{H\left(T_{n}\right)>U\left(T_{n}\right)\right\}, \quad n<m-1,
$$

having $\tau_{m-1}=T_{m-1}$. The option price is then computed using Eq. (5).

A rigorous mathematical justification and proof of the almost sure convergence of the method can be found in Clement et al. (2002).

\subsection{The stochastic grid bundling method}

SGBM is a simulation-based dynamic programming method, which first generates Monte Carlo paths, forward in time, followed by finding the optimal early-exercise policy moving backwards in time. The main difference between LSM and SGBM is that in SGBM one projects the option values onto a set of basis functions of the state variables where the distribution is analytically (or approximate) known, whereas in LSM this is not taken into account.

The discounted continuation value, $H\left(T_{n}\right)$ in Eq. (7), is computed using the law of iterated expectations, i.e.,

$$
\mathbb{E}[X \mid \mathcal{H}]=\mathbb{E}[\mathbb{E}[X \mid \mathcal{G}] \mid \mathcal{H}],
$$

where $\mathcal{H}$ is a sub- $\sigma$ algebra of $\mathcal{G}$. Using Eq. (10), the continuation value at time $T_{n}$ can be written as

$$
\begin{aligned}
H\left(T_{n}\right) & =B\left(T_{n}\right) \mathbb{E}\left[\frac{V\left(T_{n+1}\right)}{B\left(T_{n+1}\right)} \mid S\left(T_{n}\right)\right] \\
& =B\left(T_{n}\right) \mathbb{E}\left[\mathbb{E}\left[\frac{V\left(T_{n+1}\right)}{B\left(T_{n+1}\right)} \mid \zeta\left(T_{n+1}\right), S\left(T_{n}\right)\right] \mid S\left(T_{n}\right)\right],
\end{aligned}
$$

where $\zeta\left(T_{n}\right)=\left(\zeta_{1}\left(T_{n+1}\right), \ldots, \zeta_{q}\left(T_{n+1}\right)\right)^{\top}$ is a $q$-dimensional vector of regression variables, for example the $q$ first monomials

$$
\zeta_{i}\left(T_{n+1}\right)=S\left(T_{n+1}\right)^{i}, \quad i=1, \ldots, q,
$$

and where $S$ is the swap rates defined in (3). 
Writing the continuation value as in Eq. (11) decomposes the problem into two steps. The first step involves computing the inner conditional expectation,

$$
Z\left(T_{n}\right)=\mathbb{E}\left[\frac{V\left(T_{n+1}\right)}{B\left(T_{n+1}\right)} \mid \zeta\left(T_{n+1}\right), S\left(T_{n}\right)\right] .
$$

It is followed by the computation of the outer expectation,

$$
H\left(T_{n}\right)=B\left(T_{n}\right) \mathbb{E}\left[Z\left(T_{n}\right) \mid S\left(T_{n}\right)\right]
$$

With a smart choice of basis functions $\zeta$ and simulation measure, Eq. (14) can generally be computed in "closed-form". However, numerical approximations are involved in the computation of $Z\left(T_{n}\right)$ in Eq. (13).

Consider the conditional expectation without the extra conditioning on $S\left(T_{n}\right)$ as in Eq. (13),

$$
\mathbb{E}\left[\frac{V\left(T_{n+1}\right)}{B\left(T_{n+1}\right)} \mid \zeta\left(T_{n+1}\right)\right] .
$$

Equation (15) can be approximated by regressing $V\left(T_{n+1}\right) / B\left(T_{n+1}\right)$ onto the first $q<\infty$ basis functions, $\zeta_{1}, \ldots, \zeta_{q}$. For example, by using the polynomials of the conditioning function as the basis, e.g., polynomials up to order 2-4 constructed by the monomials of the explanatory variable.

But in order to compute $Z\left(T_{n}\right)$ in Eq. (13), we also need to condition $V\left(T_{n+1}\right)$ on $S\left(T_{n}\right)$, which can be done in two ways. In the first approach, with nested Monte Carlo simulation, the paths are simulated until the next time $T_{n+1}$ with $S\left(T_{n}\right)$ as the source, the option values for these paths are used to approximate Eq. (13). The fitted value of this regression will converge in mean square and probability, when the number of paths in this sub-simulation goes to infinity. However, this approach will be computationally intractable as the number of paths grows exponentially with each time step. The second approach, is to condition $V\left(T_{n+1}\right)$ on $S\left(T_{n}\right)$ and then use bundling.

Bundling as introduced by Tilley (1993) is a method to partition the state space into nonoverlapping regions, so that each point in the space can be identified to lie in exactly one of the bundled regions. The idea behind bundling is that for a large set of paths, the neighboring paths will have similar continuation values and one can therefore perform local-averaging. The key step is to construct bundles, by first generating $K$ paths, $\omega_{1}, \ldots, \omega_{K}$, of the underlying asset, $S\left(T_{n}, \omega_{k}\right)$, and bundle them at each time, $T_{n}$, into $a_{n}(K)$ nonoverlapping sets, $\mathcal{B}^{s}\left(T_{n}\right)=\left(\mathcal{B}^{1}\left(T_{n}\right)\right.$, $\left.\ldots, \mathcal{B}^{a}\left(T_{n}\right)\right)$. This is done by defining at each time, $T_{n}$, representative states $\mu_{n}^{s}$ for 
$s=1, \ldots, a_{n}(K)$. The $s$ th-bundle at time $T_{n}$ is thus defined as

$$
\begin{aligned}
H\left(T_{n}\right)= & \left\{S\left(T_{n}, \omega_{k}\right):\left\|S\left(T_{n}, \omega_{k}\right)-\mu_{n}^{s}\right\|_{2} \leq\left\|S\left(T_{n}, \omega_{k}\right)-\mu_{n}^{\ell}\right\|_{2},\right. \\
& \left.\forall 1 \leq \ell \leq a_{n}(K)\right\}
\end{aligned}
$$

for $k=1, \ldots, K$ and where $\mu_{n}^{s}$ is the mean of the points in $\mathcal{B}^{s}\left(T_{n}\right)$.

The continuation value (7) for a general path $\omega_{k}$ at time $T_{n}$ is then approximated by,

$$
\hat{H}\left(T_{n}, \omega_{k}\right)=B\left(T_{n}, \omega_{k}\right) \mathbb{E}\left[\frac{\widehat{V}\left(T_{n+1}, \omega_{k}\right)}{B\left(T_{n+1}, \omega_{k}\right)} \mid \mathcal{B}\left(T_{n}, \omega_{k}\right)\right],
$$

where bundle $\mathcal{B}\left(T_{n}, \omega_{k}\right)$ is the set of path-indices of paths that lie in the bundle containing $S\left(T_{n}, \omega_{k}\right)$.

SGBM employs a recursive bifurcation algorithm to bundle the grid points at each time step, the number of partitions, or bundles, after $p$ iterations, equals $2^{p}$. The algorithm is explained in detail in Appendix A and Fig. 1 illustrates the idea behind the bundling from simulated swap rates and continuation values, using two respectively four bundles.

As explained, SGBM computes the continuation value in two steps. First, we compute the expected option value, conditioned on a finer information set, given by Eq. (13), which is followed by the computation of the outer expectation, given by Eq. (14). Let $\mathcal{B}\left(T_{n}, \omega_{k}\right)$ denote the set of path-indices of paths that share the bundle containing the $k$ th grid point $S\left(T_{n}, \omega_{k}\right)$ at time $T_{n}$. Second, we approximate $Z$ in Eq. (13) by regressing the option values at $T_{n}$ for those paths that originate
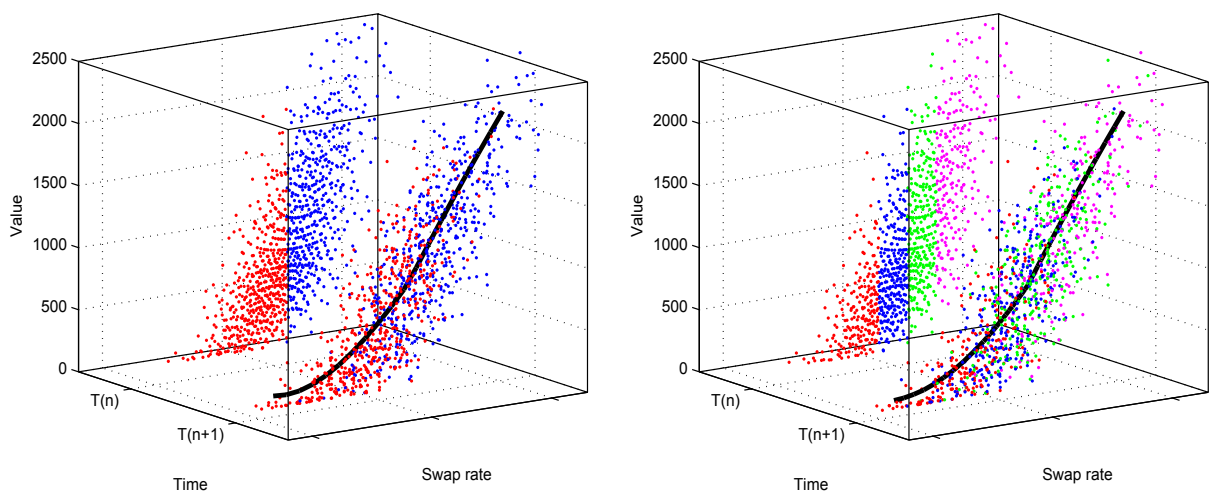

Fig. 1. Simulated continuation values as a function of the swap value for a Bermudan swaption at one of the exercise dates. Continuation values approximated by a second-order polynomial, with the swaps as basis functions. Left: Regression with two bundles. Right: Regression with four bundles. 
from the bundle containing $S\left(T_{n}, \omega_{k}\right)$, that is

$$
\hat{z}\left(T_{n}, \omega_{k}\right)=\sum_{i=1}^{q} \beta_{i, n} \zeta_{i}\left(T_{n}, \omega_{k}\right),
$$

where $k \in \mathcal{B}\left(T_{n}, \omega_{k}\right)$ so that the following residual is minimized

$$
\min _{\beta} \sum_{l \in \mathcal{B}\left(T_{n}, \omega_{k}\right)}\left(\hat{z}\left(T_{n}, \omega_{l}\right)-V\left(T_{n}, \omega_{l}\right)\right)^{2} .
$$

The continuation value for grid point $S\left(T_{n}, \omega_{k}\right)$ in bundle $\mathcal{B}\left(T_{n}, \omega_{k}\right)$ is then given by,

$$
\hat{H}\left(T_{n}, \omega_{k}\right)=B\left(T_{n}, \omega_{k}\right) \sum_{i=0}^{q} \beta_{i, n} \mathbb{E}_{T_{n}}\left[\frac{\zeta_{i}\left(T_{n+1}, \omega_{k}\right)}{B\left(T_{n+1}, \omega_{k}\right)} \mid \mathcal{B}\left(T_{n}, \omega_{k}\right)\right]
$$

Remark 3.1. SGBM requires significantly fewer paths and basis functions than LSM. The reason for this is that LSM uses the regressed continuation values to make early exercise decision directly. The quality of the early exercise policy is inaccurate when a small number of paths and basis functions are used, one therefore need a large number of paths and basis functions to reduce the regression noise. In SGBM, the regressed function is just an inner expectation. The outer expectation, which can be calculated analytically, gives the continuation value and is used for decision making. Since the regression error is normally distributed with a zero mean, the noise of outer expectation of is zero. Therefore, the continuation value surface generated by SGBM is much smoother, compared to the one generated by LSM.

Further details of SGBM, such as convergence and different bundling algorithms can be found in Jain and Oosterlee (2015).

Some of the difficulties in the pricing of the Bermudan swaptions lie in the choice of regression variables. Choosing a suitable set of explanatory variables and parametric functions is crucial. In our framework this can be considered as a combination of both art and science. An estimation of the exercise boundary close to the true boundary gives an estimated price closer to the true value. One significant problem with regression is ease of overfitting. One should not therefore use too many regression variables and high-order polynomials since they are easily affected by outliers in the simulation. One needs to focus on finding significant explanatory variables. Glasserman and Yu (2004) showed that for the simplest case of Bermudan swaptions a second-order polynomial with the underlying swap values as basis appears sufficient to obtain accurate Bermudan swaption values. 
More generally, the choice of basis functions is usually product dependent and needs to be carefully investigated for complicated Bermudan swaptions, for example for products with exotic coupons.

We conclude this section by emphasizing the choices of measures used in order to allow for efficient simulation. The $T$-forward measure, with corresponding expectation $\mathbb{E}^{T}$ and the $T$-maturity zero-coupon bond $P(t, T)$ as the numeraire have the advantage that it allows for decoupling the payoff $V(T)$ from the numeraire and to take out the discount factor from the expectation, i.e.,

$$
V(t)=B(t) \mathbb{E}_{t}\left[\frac{V(T)}{B(T)}\right]=P(t, T) \mathbb{E}_{t}^{T}[V(T)] .
$$

One benefit, however, of the spot measure compared to the $T$-forward measure is that the numeraire asset $B(t)$ is alive throughout the tenor and therefore, allows for simulating paths irrespective of tenor. We employ hybrid measures to obtain efficient Monte Carlo simulation. The inner expectation is simply approximated by regression calculated in the spot measure. To express the outer expectation in closed form, we compute the expectation under the $T$-forward measure. Since the spot measure $\mathbb{Q}^{B}$ coincides with the $T_{n+1}$-forward measure $\mathbb{Q}^{T_{n+1}}$ over the interval $\left[t_{n}, t_{n+1}\right]$ this allows us to write the continuation value for grid point $S\left(T_{n}, \omega_{k}\right)$ in bundle $\mathcal{B}\left(T_{n}, \omega_{k}\right)$ as,

$$
\begin{aligned}
\widehat{\mathrm{H}}\left(T_{n}, \omega_{k}\right) & =B\left(T_{n}, \omega_{k}\right) \mathbb{E}\left[\frac{\sum_{i=0}^{q} \beta_{i, n} \zeta_{i}\left(T_{n+1}, \omega_{k}\right)}{B\left(T_{n+1}, \omega_{k}\right)} \mid \mathcal{B}\left(T_{n}, \omega_{k}\right)\right] \\
& =P\left(T_{n}, T_{n+1}, \omega_{k}\right) \mathbb{E}^{T_{n+1}}\left[\sum_{i=0}^{q} \beta_{i, n} \zeta_{i}\left(T_{n+1}, \omega_{k}\right) \mid \mathcal{B}\left(T_{n}, \omega_{k}\right)\right] \\
& =P\left(T_{n}, T_{n+1}, \omega_{k}\right) \sum_{i=0}^{q} \beta_{i, n} \mathbb{E}^{T_{n+1}}\left[\zeta_{i}\left(T_{n+1}, \omega_{k}\right) \mid \mathcal{B}\left(T_{n}, \omega_{k}\right)\right] .
\end{aligned}
$$

By this we can efficiently simulate the exposure. We refer to (Andersen and Piterbarg, 2010, Chapter 4) for additional information on available fixed-income probability measures.

Remark 3.2. Valuation of Bermudan swaptions with American Monte Carlo techniques such as LSM and SGBM requires an estimation of the exercise boundary. The option can then be seen as a barrier option (knock-in) with the estimated exercise boundary as the barrier.

\subsubsection{Algorithm for lower bound}

For clarity we summarize the steps of the complete SGBM pricing process for the Bermudan swaptions. We first simulate a first pass with $K_{1}$ paths and then estimate 
an exercise policy. Second, we simulate a second pass with $K_{2}$ paths using the exercise policy estimated in the first pass. Usually $K_{2} \approx 10,000-100,000$ and $K_{1} \approx K_{2} / 4$.

\section{FIRST PASS: Exercise Policy}

1. Generate $K_{1}$ paths $\omega_{1}, \ldots, \omega_{K_{1}}$, using (1). Each $\omega_{k}$ represents one simulated path of all core LIBOR rates.

2. For each path $\omega_{k}$ and time $T_{n}$, for $k=1, \ldots, K_{1}$ and $n=1, \ldots, N-1$, calculate the numeraire $B\left(T_{n}, \omega_{k}\right)$, swap rates $S\left(T_{n}, \omega_{k}\right)$ and exercise values $U\left(T_{n}, \omega_{k}\right)$.

3. Compute the option value for the grid points at the terminal time $T_{m-1}$,

$$
V\left(T_{m-1}\right)=\max \left(U\left(T_{m-1}\right), 0\right) .
$$

4. For each $n=m-2 \ldots, 1$

(a) Bundle the grid points at $T_{n-1}$, into $a$ distinct bundles (except at $T_{0}$, where there is only one point and hence only one bundle corresponding to $S\left(T_{0}\right)$ ) using the bundling algorithm in Appendix A.

(b) Compute the regression functions, $Z_{n}^{s}, s=1, \ldots, a$, as given by Eq. (18), using the option values at $T_{n}$ for the paths originating from the $s$ th bundle, $\mathcal{B}^{s}\left(T_{n-1}\right)$, at $T_{n-1}$.

(c) Compute the continuation value for the grid points in the sth bundle at $T_{n-1}$, using Eq. (19), for those paths for which $S\left(T_{n}, \omega_{k}\right)$ belongs to the bundle $\mathcal{B}^{s}\left(T_{n-1}\right)$, for $s=1, \ldots, a$.

(d) Compute the option values at $T_{n-1}$, as

$$
\widehat{V}\left(T_{n}\right)=\max \left(U\left(T_{n}\right), H\left(T_{n}\right)\right) .
$$

5. The option value, $\widehat{V}\left(T_{0}\right)$, at $T_{0}$, is defined to be the direct estimator value.

\section{SECOND PASS: Lower Bound}

1. In order to compute lower bounds and an unbiased price, generate a fresh set of $K_{2}$ paths, $\omega_{1}^{\prime}, \ldots, \omega_{K_{2}}^{\prime}$, and bundle at each time step, using the same algorithm as in the first pass.

2. The continuation values for the grid points in bundle $s$, at time step $T_{n-1}$, are computed using the $Z_{n}^{s}$ function, obtained for the direct estimator. The option is exercised when the continuation value is less than the immediate payoff. The lower bound can then be computed by determining the earliest time to exercise at each path, $\widehat{\tau}\left(\omega_{k}^{\prime}\right)=\min \left\{T_{n}: H\left(T_{n}, \omega_{k}^{\prime}\right)<U\left(T_{n}, \omega_{k}^{\prime}\right)\right\}$. The lower bound of 
the option value is given by,

$$
\underline{V}\left(T_{0}\right)=\frac{1}{K_{2}} \sum_{k=1}^{K_{2}} \frac{B\left(T_{0}, \omega_{k}^{\prime}\right)}{B\left(\hat{\tau}, \omega_{k}^{\prime}\right)} U\left(\hat{\tau}, \omega_{k}^{\prime}\right) .
$$

Remark 3.3. In the case of performance calculation issues, the direct estimate generated from the first pass will often be close to the lower bound values generated from the second pass. In this case, one can neglect the second pass but should keep in mind that the estimated values are biased in an unknown direction.

Remark 3.4. One should also keep in mind that both LSM and SGBM are lower bound methods, basically because the conditional expectation is approximated by a regression technique that projects the high dimensional continuation value onto a limited set of regression variables. The approximation can often be improved, for example, by having a richer and better set of regression variables, but with the risk of overfitting.

\subsection{Upper bound using dual formulation}

One problem with the lower bound algorithm presented in the previous section is to determine how close the generated option prices are to the true value. One way to determine its goodness it to simulate both lower and upper bounds of the option values, the closer they are to each other the better. Haugh and Kogan (2001) and Rogers (2002) independently proposed the dual formulation for Bermudan options, later extended to the primal-dual simulation algorithm in Andersen and Broadie (2004). The primal problem is given by Eq. (4), for an arbitrary adapted super-martingale process $M(t)$ we have that,

$$
\begin{aligned}
V\left(T_{0}\right) & =\sup _{\tau \in \mathcal{T}} \mathbb{E}_{T_{0}}\left[\frac{U(\tau)}{B(\tau)}\right] \\
& =\sup _{\tau \in \mathcal{T}} \mathbb{E}_{T_{0}}\left[\frac{U(\tau)}{B(\tau)}+M(\tau)-M(\tau)\right] \\
& =M(0)+\sup _{\tau \in \mathcal{T}} \mathbb{E}_{T_{0}}\left[\frac{U(\tau)}{B(\tau)}-M(\tau)\right] \\
& \leq M(0)+\mathbb{E}_{T_{0}}\left[\sup _{\tau \in \mathcal{T}}\left(\frac{U(\tau)}{B(\tau)}-M(\tau)\right)\right] .
\end{aligned}
$$

The inequality follows from the fact $M(t)$ is a super-martingale. The dual formulation of the option pricing problem is then to minimize the upper bound with 
respect to all adapted super-martingale processes $\mathcal{K}$, that is,

$$
\bar{V}_{0}=\inf _{M \in \mathcal{K}}\left\{M(0)+\mathbb{E}_{T_{0}}\left[\sup _{\tau \in \mathcal{T}}\left(\frac{U(\tau)}{B(\tau)}-M(\tau)\right)\right]\right\} .
$$

Haugh and Kogan (2001) showed that when the super-martingale process $M(t)$ in Eq. (22) coincides with the discounted option value process $V(t) / B(t)$, the upper bound $\bar{V}_{0}$ equals the true value. This suggests that a tight upper bound can be obtained by approximation $\widehat{V}(t)$, when defining $M(t)$ such that when the approximate option price $\widehat{V}(t)$ coincides with the exact price $V(t), M(t)$ equals the discounted process $V(t) / B(t)$. An obvious choice for $M(t)$ is then given by

$$
M\left(T_{n+1}\right)-M\left(T_{n}\right)=\frac{\widehat{V}\left(T_{n+1}\right)}{B\left(T_{n+1}\right)}-\frac{\widehat{V}\left(T_{n}\right)}{B\left(T_{n}\right)}-\mathbb{E}_{T_{n}}\left[\frac{\widehat{V}\left(T_{n+1}\right)}{B\left(T_{n+1}\right)}-\frac{\widehat{V}\left(T_{n}\right)}{B\left(T_{n}\right)}\right],
$$

for $M\left(T_{0}\right)=\widehat{V}\left(T_{0}\right)$. Equation (24) can also be written as

$$
M\left(T_{n+1}\right)-M\left(T_{n}\right)=\frac{\widehat{V}\left(T_{n+1}\right)}{B\left(T_{n+1}\right)}-\mathbb{E}_{T_{n}}\left[\frac{\widehat{V}\left(T_{n+1}\right)}{B\left(T_{n+1}\right)}\right] .
$$

Then the upper bound, $\bar{V}_{0}$, corresponding to Eq. (24) is given by

$$
\bar{V}(0)=\widehat{V}(0)+\Delta \geq V(0),
$$

where the duality gap $\Delta$ is defined as

$$
\Delta=\max _{n}\left(\frac{U\left(T_{n}\right)}{B\left(T_{n}\right)}-M\left(T_{n}\right)\right), \quad n=1, \ldots, m-1 .
$$

Generally, upper bounds algorithm as e.g., in Andersen and Broadie (2004) require nested Monte Carlo simulation and the quality of the upper bound produced by the algorithm depends on the quality of the estimated exercise policy in the first pass, better policy gives tighter upper-lower bounds. This makes it computational expensive and requires in worst cases a workload of $K \times K_{\text {nest }} \times m^{2}$ operations, where $K$ is the number of outer simulations, $K_{\text {nest }}$ the number of nested simulations, and $m$ the number of exercise dates. The workload is often less than this because the nested simulation can be stopped whenever the contract is exercised. This in comparison with the workload in the second pass where an exercise policy already is given and where the lower bound simulation has a workload of $K \times m$. This has further been improved by Broadie and Cao (2008) who showed that nested simulations are not needed on dates where it is sub-optimal to exercise the 
option, which can lead to reduced workload, especially for out-of-the-money options. When the policy obtained from LSM is used, a sub-simulation with $K_{\text {nest }}$ sub-paths is required. $K_{\text {nest }} \approx 100$ is often sufficient to find upper bounds with sufficient quality. Moreover, the upper bound bias introduced from the Monte Carlo simulation is positive and a decreasing function in the number of nested simulations.

We conclude this section by emphasizing one important remark allowing one to avoid nested Monte Carlo simulations for upper bound values when estimating the exercise policy using SGBM.

Remark 3.5. For pure regression-based algorithms like LSM, Eq. (25) cannot be estimated directly by regression since it will introduce an unknown bias and therefore destroys the martingale property of $M$ and the inequality in Eq. (22). Therefore, one has to rely on nested Monte Carlo simulations to obtain an upper bound when LSM is used. But, as mentioned in Remark 3.1, the regressed function in SGBM is just the inner expectation, and it is not used for decision-making. The outer expectation can be computed in closed form and we can therefore calculate the upper bounds without nested simulations. This reduces the workload of the upper bounds significantly, to the workload of the second pass and therefore we can obtain a speed-up factor of $K_{\text {nest }}$. The computational time for SGBM is comparable to Longstaff and Schwartz (2001).

\subsubsection{Algorithm for upper bound}

We summarize the simulation procedure for obtaining duality-based upper bounds via SGBM below (once the optimal exercise policy has been obtained). Let $\hat{H}\left(T_{n}\right)$ be the holding value estimated from the exercise strategy $\eta$, given by the simulation in the first pass. The upper bound can then be obtained by the following algorithm.

\section{THIRD PASS: Upper Bound}

1. Simulate $K_{u}$ paths $\omega_{1}, \ldots, \omega_{K_{U}}$

2. For each exercise time $T_{n}$ and each path $\omega_{k}$, compute $\hat{H}\left(T_{n}, \omega_{k}\right)$ and $B\left(T_{n}, \omega_{k}\right)$, and update $M\left(T_{n}, \omega_{k}\right)$ in Eq. (28).

(a) Approximate $\hat{H}\left(T_{n}, \omega_{k}\right) / B\left(T_{n}, \omega_{k}\right)$ using Eq. (17). ${ }^{2}$

\footnotetext{
${ }^{2}$ In order to have an accurate approximation of $H\left(T_{n}, \omega_{k}\right)$, when the policy obtained from LSM is used, a sub-simulation with $K_{\text {nest }}$ sub-paths is required. $\hat{H}\left(T_{n}, \omega_{k}\right)$ represents the discounted average cashflows from these paths when they are exercised following the policy obtained in the first pass.
} 
3. For each path $\omega_{K}$, compute the pathwise duality gaps, as follows:

$$
\widehat{D}\left(\omega_{k}\right)=\max _{n}\left(\frac{U\left(T_{n}, \omega_{k}\right)}{B\left(T_{n}, \omega_{k}\right)}-M\left(T_{n}, \omega_{k}\right)\right), \quad n=1, \ldots, m .
$$

4. Estimate the upper bound given by Eq. (28) as

$$
\widehat{\Delta}=\frac{1}{K_{U}} \sum_{k=1}^{K_{U}} \hat{D}\left(\omega_{k}\right) \text {. }
$$

\section{Numerical Results}

In this section, we study the performance of SGBM for lower and upper bound values by means of numerical experiments. For a consistency check we use the same setup and reproduce the results in Andersen (1999) and Andersen and Piterbarg (2010).

\subsection{Bermudan swaption prices}

For the continuation value in LSM and SGBM, we use a second-order polynomial with the swap rate as the basis. The swap rate moments in Eq. (19) can, for example, with high accuracy be calculated by the convexity adjustment approach in Belomestny et al. (2009).

We use the bundling scheme described in Section Appendix A, with eight bundles and the same number of bundles at each time step, except at time $T_{0}$, where there is only one point, $S\left(T_{0}\right)$.

We consider Bermudan swaptions on three months LIBORS $(\tau=0.25)$ with $10 \%$ spot rate level and with two different volatility settings. First, a one-factor LMM with fixed volatility, $\lambda_{n}(t)=0.2$ for all $n$ and $t$. Second, more realistically, a two-factor LMM, with a time-to-maturity dependent volatility of the form ${ }^{3}$

$$
\lambda_{n}(t)=\left[0.15,0.15-\sqrt{0.009\left(T_{n}-t\right)}\right]^{\top} .
$$

We report values obtained from the second pass. First, we simulate a first pass with 10,000 seeds using an antithetic Monte Carlo random number generator and then estimate the exercise policy for both LSM and SGBM. Subsequently, we simulate 20,000 second pass paths with a quasi Monte Carlo random number generator (e.g., Sobol sequence) with the previously obtained exercise policy to estimate the

\footnotetext{
${ }^{3}$ Usually, a one-factor LMM already accounts for more than $98 \%$ and the two-factor for more than $99.5 \%$ of the overall variance.
} 
unbiased Bermudan swaption value. These two steps are repeated iteratively $K^{\prime}=$ 100 times with different seeds in the first simulation, to remove the overall influence of the first simulation. The prices are reported in basis points, with the notional $\mathcal{N}=10,000$ and the numbers in parentheses are sample standard deviations.

Duality-based upper bounds, together with the lower bound computed using the path estimator give valid confidence intervals within which the true option price lies. The $100(1-\gamma) \%$ confidence interval is constructed as

$$
\left[\underline{V}_{0}\left(T_{0}\right)-q_{\gamma / 2} \frac{\hat{s}_{L}}{\sqrt{K^{\prime}}}, \bar{V}_{0}\left(T_{0}\right)+q_{\gamma / 2} \frac{\hat{s}_{H}}{\sqrt{K^{\prime}}}\right],
$$

where $\hat{s}_{L}$ is the sample standard deviation for the path estimator and $\hat{s}_{H}$ is the sample standard deviation for the duality-based upper bound estimator, and $q_{\gamma / 2}$ the normal distributed quantile function.

Tables 1 and 2 report the lower bound value estimates for the Bermudan swaption via LSM and SGBM, the duality gap and 95\%-confidence interval with one-factor, respectively two-factor LMM. Our reported values for SGBM differ at most 3 bps compared to the reported values in Andersen and Piterbarg (2010). The computational time for SGBM is roughly the same as for the LSM. The first conclusion, the standard deviation for SGBM lower bounds is much smaller than

Table 1. Lower bound estimate of Bermudan payer swaptions in a one-factor LMM. Prices are in basis points and standard deviations within parentheses.

\begin{tabular}{lrrrrrrrr}
\hline Type & Strike $(\%)$ & \multicolumn{2}{c}{ LSM lower } & \multicolumn{2}{c}{ SGBM lower } & $\widehat{\Delta}_{\text {SGBM }}$ & $\widehat{\Delta}_{\text {AP }}$ & $95 \%$ CI \\
\hline $15 \mathrm{M} / 3 \mathrm{M}$ & 8 & 184.61 & $(0.01)$ & 184.62 & $(0.00)$ & 0.0022 & 0.02 & $184.62-184.63$ \\
$15 \mathrm{M} / 3 \mathrm{M}$ & 10 & 49.11 & $(0.01)$ & 49.11 & $(0.00)$ & 0.0008 & 0.02 & $49.111-49.114$ \\
$15 \mathrm{M} / 3 \mathrm{M}$ & 12 & 8.73 & $(0.02)$ & 8.73 & $(0.00)$ & 0.0001 & 0.004 & $8.7322-8.7346$ \\
$3 \mathrm{Y} / 1 \mathrm{Y}$ & 8 & 355.08 & $(0.08)$ & 355.06 & $(0.02)$ & 0.0133 & 0.07 & $355.05-355.07$ \\
$3 \mathrm{Y} / 1 \mathrm{Y}$ & 10 & 157.13 & $(0.11)$ & 157.45 & $(0.03)$ & 0.0030 & 0.2 & $157.45-157.46$ \\
$3 \mathrm{Y} / 1 \mathrm{Y}$ & 12 & 60.96 & $(0.07)$ & 60.97 & $(0.02)$ & 0.0011 & 0.04 & $60.97-60.98$ \\
$6 \mathrm{Y} / 1 \mathrm{Y}$ & 8 & 806.61 & $(0.41)$ & 808.11 & $(0.08)$ & 0.0186 & 0.23 & $808.09-808.14$ \\
$6 \mathrm{Y} / 1 \mathrm{Y}$ & 10 & 415.35 & $(0.82)$ & 418.58 & $(0.13)$ & 0.0088 & 0.63 & $418.55-418.61$ \\
$6 \mathrm{Y} / 1 \mathrm{Y}$ & 12 & 212.13 & $(0.48)$ & 214.16 & $(0.12)$ & 0.0041 & 0.33 & $214.13-214.19$ \\
$11 \mathrm{Y} / 1 \mathrm{Y}$ & 8 & 1377.00 & $(1.07)$ & 1383.10 & $(0.26)$ & 0.0307 & 1.3 & $1383.00-1383.10$ \\
$11 \mathrm{Y} / 1 \mathrm{Y}$ & 10 & 805.93 & $(1.00)$ & 811.13 & $(0.23)$ & 0.0188 & 1.3 & $811.08-811.20$ \\
$11 \mathrm{Y} / 1 \mathrm{Y}$ & 12 & 495.16 & $(0.69)$ & 499.20 & $(0.27)$ & 0.0120 & 0.7 & $499.15-499.27$ \\
$6 \mathrm{Y} / 3 \mathrm{Y}$ & 8 & 493.91 & $(0.15)$ & 494.12 & $(0.04)$ & 0.0235 & 0.08 & $494.11-494.15$ \\
$6 \mathrm{Y} / 3 \mathrm{Y}$ & 10 & 291.84 & $(0.22)$ & 293.03 & $(0.05)$ & 0.0092 & 0.65 & $293.02-293.05$ \\
$6 \mathrm{Y} / 3 \mathrm{Y}$ & 12 & 169.22 & $(0.19)$ & 169.79 & $(0.04)$ & 0.0040 & 0.53 & $169.79-169.80$ \\
\hline
\end{tabular}


P. Karlsson, S. Jain \& C. W. Oosterlee

Table 2. Lower bound estimate of Bermudan payer swaptions in a two-factor LMM. Prices are in basis points and standard deviations within parentheses.

\begin{tabular}{lcccccccc}
\hline Type & Strike $(\%)$ & \multicolumn{2}{c}{ LSM lower } & \multicolumn{2}{c}{ SGBM lower } & $\widehat{\Delta}_{\text {SGBM }}$ & $\widehat{\Delta}_{\text {AP }}$ & $95 \%$ CI \\
\hline $15 \mathrm{M} / 3 \mathrm{M}$ & 8 & 183.83 & $(0.01)$ & 183.83 & $(0.00)$ & 0.0003 & 0.05 & $183.83-183.83$ \\
$15 \mathrm{M} / 3 \mathrm{M}$ & 10 & 42.17 & $(0.02)$ & 42.24 & $(0.02)$ & 0.0009 & 0.06 & $42.238-42.247$ \\
$15 \mathrm{M} / 3 \mathrm{M}$ & 12 & 5.21 & $(0.01)$ & 5.22 & $(0.01)$ & 0.0001 & 0.01 & $5.2183-5.2204$ \\
$3 \mathrm{Y} / 1 \mathrm{Y}$ & 8 & 339.15 & $(0.05)$ & 339.35 & $(0.02)$ & 0.0102 & 0.4 & $339.34-339.36$ \\
$3 \mathrm{Y} / 1 \mathrm{Y}$ & 10 & 125.12 & $(0.06)$ & 125.58 & $(0.02)$ & 0.0024 & 0.7 & $125.57-125.58$ \\
$3 \mathrm{Y} / 1 \mathrm{Y}$ & 12 & 35.76 & $(0.05)$ & 35.87 & $(0.02)$ & 0.0004 & 0.2 & $35.866-35.875$ \\
$6 \mathrm{Y} / 1 \mathrm{Y}$ & 8 & 747.23 & $(0.19)$ & 751.88 & $(0.06)$ & 0.0128 & 3.7 & $751.86-751.9$ \\
$6 \mathrm{Y} / 1 \mathrm{Y}$ & 10 & 315.73 & $(0.40)$ & 319.18 & $(0.10)$ & 0.0054 & 5.0 & $319.16-319.21$ \\
$6 \mathrm{Y} / 1 \mathrm{Y}$ & 12 & 126.41 & $(0.31)$ & 129.14 & $(0.08)$ & 0.0020 & 2.6 & $129.12-129.16$ \\
$11 \mathrm{Y} / 1 \mathrm{Y}$ & 8 & 1237.80 & $(0.63)$ & 1253.40 & $(0.20)$ & 0.0191 & 18.1 & $1253.4-1253.5$ \\
$11 \mathrm{Y} / 1 \mathrm{Y}$ & 10 & 610.34 & $(0.65)$ & 628.93 & $(0.26)$ & 0.0142 & 20.8 & $628.88-628.99$ \\
$11 \mathrm{Y} / 1 \mathrm{Y}$ & 12 & 322.55 & $(0.67)$ & 335.18 & $(0.17)$ & 0.0071 & 14.8 & $335.15-335.22$ \\
$6 \mathrm{Y} / 3 \mathrm{Y}$ & 8 & 444.83 & $(0.16)$ & 446.15 & $(0.03)$ & 0.0194 & 0.8 & $446.14-446.17$ \\
$6 \mathrm{Y} / 3 \mathrm{Y}$ & 10 & 225.67 & $(0.14)$ & 227.24 & $(0.04)$ & 0.0054 & 1.2 & $227.23-227.25$ \\
$6 \mathrm{Y} / 3 \mathrm{Y}$ & 12 & 106.16 & $(0.11)$ & 107.27 & $(0.03)$ & 0.0019 & 0.8 & $107.26-107.27$ \\
\hline
\end{tabular}

the ones obtained from LSM. On average, the ratio of variance of LSM and SGBM is around 16, meaning that on average, one would need 16 times fewer Monte Carlo seeds in order to obtain the same pricing accuracy. The second conclusion, the duality gap $\widehat{\Delta}_{\text {SGBM }}$ obtained by SGBM is significantly smaller than the duality gap $\widehat{\Delta}_{\mathrm{AP}}$ as reported in Andersen and Piterbarg (2010). As one can observe we obtain significantly smaller duality gaps, the largest duality gap for the one-factor LMM is 0.0307 basis points, compared to 1.3 basis points in Andersen and


Fig. 2. Exercise boundary for LSM and SGBM with bundles, $2^{q}$, for $q=0,1,2,3$ for a $3 \mathrm{Y} / 1 \mathrm{Y}$ Bermudan payer swaptions in a one-factor LMM. 

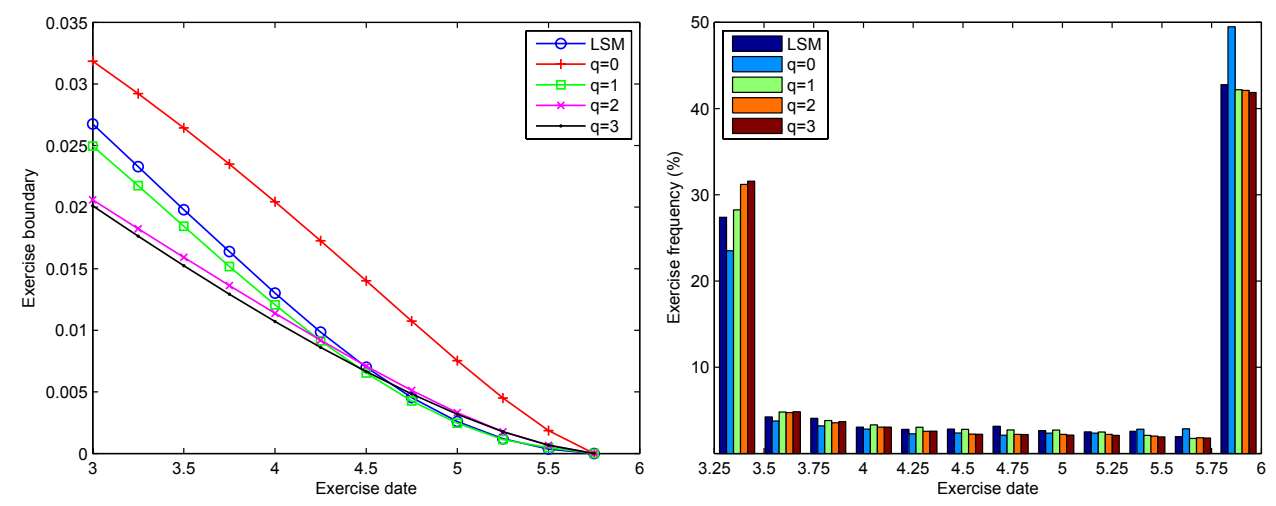

Fig. 3. Exercise boundary for LSM and SGBM with bundles, $2^{q}$, for $q=0,1,2,3$ for a $6 \mathrm{Y} / 3 \mathrm{Y}$ Bermudan payer swaptions in a two-factor LMM.

Broadie (2004). The conclusion here is that the duality gap obtained by SGBM gives rise to really tight lower-upper bounds.

Figures 2 and 3 illustrate the exercise boundary and the exercise frequency for the one-and two-factor LMM, respectively. The pictures demonstrate the sensitivity of the product regarding the exercise policy. We can clearly see that the Bermudan swaption is mostly canceled directly at the first or last exercise date. The figures also demonstrate that the stopping times are not very different from

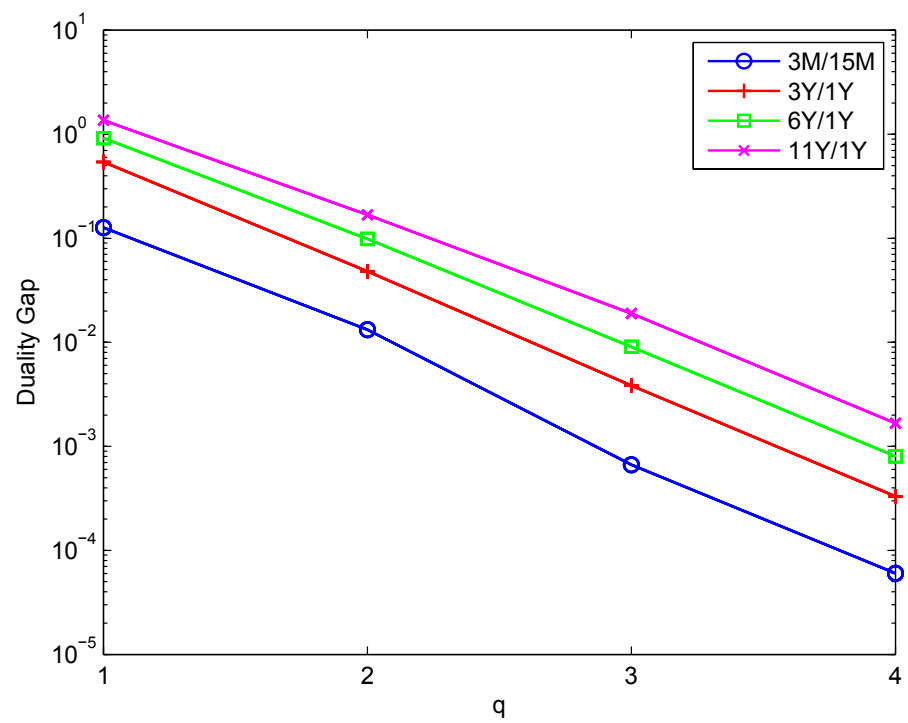

Fig. 4. The duality gap from SGBM with varying numbers of bundles, $2^{q}$, for $q=1,2,3,4$ for Bermudan payer swaptions in a one-factor LMM. 
each other, although the few scenarios in which SGBM in comparison with LSM exercises earlier have a significant effect on the price. This is the reason why the SGBM prices are superior to the LSM prices in these tests and why SGBM demonstrates tight lower-upper bounds.

\subsection{The effect of number of bundles}

In this section, we study how the number of bundles affect the lower-upper bound values and the duality gap. In particular, we study Bermudan swaptions with the same setup as in the previous section for a $10 \%$ coupon with $2^{p}$ bundles for $p=1,2,3,4$. The duality gap is illustrated in Fig. 4 . We observe an almost loglinear relationship between the duality gap and the number of bundles. Increasing the number of bundles will make the duality gap much smaller.

\section{Conclusion}

This paper presented the application of the SGBM for approximating the values of Bermudan style options on the LMM by simulation. SGBM is a regression-based Monte Carlo method where the continuation value is projected onto a space where the distribution is known. In the method, a practical bundling algorithm is employed which completes the algorithm and performs very well for the test cases considered. We also demonstrate how to obtain upper bounds without the need for nested Monte Carlo simulations as generally required for regression-based methods. The upper-lower bounds obtained by SGBM are much tighter compared to the bounds obtained by traditional methods. We illustrate SGBM's performance using a number of realistic examples. The computational time for the method is comparable to Longstaff and Schwartz (2001), but a higher accuracy is achieved as demonstrated by the reduced Monte Carlo variance. The SGBM method is easy to implement and accurate. Variance reduction, based on iterated conditioning, in combination with the bundling technique form the necessary ingredients for accurate Bermudan swaptions valuation with a relative small number of paths and basis functions. One should however keep in mind that we need to know the conditional expected value of the basis functions.

\section{Acknowledgment}

The work of this paper was carried out while Patrik Karlsson was a PhD Student at Department of Economics, Lund University, Sweden, and held a visiting scholar position at the CWI-Centrum Wiskunde \& Informatica, Amsterdam, The Netherlands, November 2012 through February 2013. He wishes to thank Nada 
Mitrovic, Cornelis W. Oosterlee and the Scientific Computing group for their hospitality.

\section{Appendix A. Bundling}

Suppose we need to bundle $K_{s}$ grid points at epoch $T_{n}$, given by $S\left(T_{n}, \omega_{k}\right)$, where $k=1, \ldots, K_{s}$. The following steps are performed recursively.

(1) Compute the mean of the given set of grid points,

$$
\mu_{n}^{s}=\frac{1}{K_{s}} \sum_{k=1}^{K_{s}} S\left(T_{n}, \omega_{k}\right) .
$$

(2) Bundling the grid points is performed by dividing the grid points into two groups, depending on whether the asset price for the grid point is greater or less than the mean of the asset prices for the given set of grid points:

$$
\begin{aligned}
& \mathcal{B}^{1}\left(T_{n}, \omega_{k}\right)=\mathbf{1}\left(S\left(T_{n}, \omega_{k}\right)>\mu_{n}^{s}\right), \\
& \mathcal{B}^{2}\left(T_{n}, \omega_{k}\right)=\mathbf{1}\left(S\left(T_{n}, \omega_{k}\right) \leq \mu_{n}^{s}\right),
\end{aligned}
$$

for $k=1, \ldots, K_{s} \cdot \mathcal{B}^{1}\left(T_{n}, \omega_{k}\right)$ returns 'true', when the asset price $S\left(T_{n}, \omega_{k}\right)$ is greater than the mean, $\mu_{n}^{s}$ and belongs to bundle $1 . \mathcal{B}^{2}\left(T_{n}, \omega_{k}\right)$ returns 'true', if it less than the mean and belongs to bundle 2 . Formally, $\mathcal{B}^{s}\left(T_{n}, \omega_{k}\right)$ returns 'true', if the grid point $S\left(T_{n}, \omega_{k}\right)$ belongs to bundle $s$.

(3) Bundles $\mathcal{B}^{1}\left(T_{n}\right)$ and $\mathcal{B}^{2}\left(T_{n}\right)$, can be split again, returning to step 1.

\section{References}

Andersen, L (1999). A simple approach to the pricing of Bermudan swaptions in the multifactor LIBOR market model, The Journal of Computational Finance, 3, 5-32.

Andersen, L and M Broadie (2004). A primal-dual simulation algorithm for pricing multidimensional American options, Management Science, 50, 1222-1234.

Andersen, L and V Piterbarg (2010). Interest Rate Modeling: Models, Products and Risk Management. London: Atlantic Financial Press.

Belomestny, D, A Kolodko and J Schoenmakers (2009). Pricing CMS spreads in the LIBOR market model, International Journal of Theoretical and Applied Finance, 13 45-62.

Black, F (1976). The pricing of commodity contracts, Journal of Financial Econometrics, 3, 167-179.

Brace, A, D Gatarek and M Musiela (1997). The market model of interest rate dynamics, Mathematical Finance, 7, 127-154. 
Broadie, M and M Cao (2008). Improved lower and upper bound algorithms for pricing American options by simulation, Quantitative Finance, 8, 845-861.

Clement, E, D Lamberton and P Protter (2002). An analysis of a least squares regression method for american option pricing, Finance and Stochastics, 6, 449-471.

de Graaf, C, Q Feng, D Kandhai and CW Oosterlee (2014). Efficient computation of exposure profiles for counterparty credit risk, to appear in International Journal of Theoretical and Applied Finance.

Glasserman, P and B Yu (2004). Number of paths versus number of basis functions in American option pricing, Annals of Applied Probability, 14, 2090-2119.

Haugh, M and L Kogan (2001). Pricing American options: A duality approach, Opertions Research, 52, 258-270.

Jain, S and CW Oosterlee (2012a). Pricing high-dimensional Bermudan options using stochastic grid method, Internation Journal of Computer Mathematics, 89, 11861211.

Jain, S and CW Oosterlee (2015). The stochastic grid bundling method: Efficient pricing of multidimensional Bermudan options and the Greeks, Applied Mathematics and Computation, 269, 412-431.

Jamshidian, F (1997). LIBOR and swap market models and measures, Finance and Stochastics, 1, 293-330.

Jin, X, HH Tana and J Sun (2007). A state-space partitioning method for pricing highdimensional American-style options, Mathematical Finance, 17, 399-426.

Longstaff, FA and ES Schwartz (2001). Valuing American options by simulation: A simple least-squares approach, Review of Financial Studies, 3, 113-147.

Milstein, GN and MV Tretyakov (2009). Practical variance reduction via regression for simulating diffusions, SIAM Journal of Numerical Analysis, 47, 887-910.

Miltersen, K, K Sandmann and D Sondermann (1997). Closed form solutions for term structure derivatives with log-normal interest rates, Journal of Finance, 52, 409-430.

Rogers, L (2002). Monte Carlo valuation of American options, Mathematical Finance, 12, 271-286.

Tilley, JA (1993). Valuing American options in a path simulation model, Transactions of the Society of Actuaries, 45, 83-104. 